\title{
REPARTITION ET ETAGEMENT DE L'HABITAT CHEZ LES PLANAIRES TRICLADES D'EAU DOUCE EN PROVENCE OCCIDENTALE
}

\author{
par Pierre LegieR ${ }^{1}$.
}

En regroupant toutes les observations faites sur la répartition des Planaires à l'intérieur d'un cours d'eau, il semble que l'étagement des espèces, en Europe, puisse être schématisé de la façon suivante. La région des sources est peuplée par Crenobia alpina; Polycelis felina occupe la zone des ruisselets collecteurs; le cours moyen du ruisseau héberge Dugesia gonocephala alors que plus en aval, Polycelis nigra fait son apparition (fig. $2 \mathrm{~A}$ ).

En Provence, la séquence est toujours incomplète : Crenobia alpina n'est jamais présente et Polycelis felina est souvent absente des cours d'eau. Par contre, la présence dans cette région de Dugesia tigrina, espèce introduite, pose le problème de leur place dans la séquence. De plus, la faible longueur de certains cours d'eau amène le rapprochement d'espèces ordinairement séparées, telles Polycelis felina et Polycelis nigra (sensu lato).

\section{1. - Répartition géographique (fig. 1).}

Mes récoltes ont été effectuées dans une trentaine de cours d'eau échelonnés entre 0 et $600 \mathrm{~m}$ d'altitude. La région prospectée s'étend, d'une part, au sud d'une ligne Cavaillon-Draguignan et, d'autre part, depuis l'étang de Berre à l'ouest jusqu'au lac de Carces à l'est.

1. Laboratoire de Biologie Animale (Ecologie), Université de Provence, SaintJérôme, Traverse de la Barasse, Marsellle (13e).

2. Le groupe Polycelis nigra (sensu lato) renferme deux espèces très voisines morphologiquement: nigra d'une part, tenuis d'autre part. Leur séparation taxonomique a été faite sur un matériel prélevé en Provence par Lascombe et Pattee (in litt.). Dans cette région, les deux espèces affines se trouvent réparties dans un secteur géographique limité (depuis l'étang de Berre jusqu'au Luberon). Pour la présente étude, j'ai préféré les maintenir au sein d'un même groupe d'espèces car elles ne présentent pas de différenciation écologique. 
Cette région renferme trois des quatre espèces qui constituent la séquence précédemment citée; il faut y ajouter Dugesia subtentaculata, Dugesia lugubris, Dugesia tigrina et Dendrocoelum lacteum.

La répartition géographique de ces sept espèces est fonction de leur plus ou moins grande eurythermie et de leur réhophilie plus ou moins accusée (LEgIER 1969 a). Les espèces qui, dans les conditions naturelles, semblent les plus eurythermes : Dugesia lugubris, Polycelis nigra, Dendrocoelum lacteum et, à un dégré moindre, Dugesia gonocephala, possèdent des aires de répartition assez étendues : toute la région prospectée pour les trois premières, toute la région prospectée sauf la zone littorale pour la dernière.

Par contre, deux espèces : Polycelis felina et Dugesia subtentaculata ont des aires de répartition réduites : la première habite toute la région nord-est $d u$ territoire et étend son aire en direction du massif de la Sainte-Baume, la seconde peuple la zone littorale et remonte vers le nord-est par la dépression d'Aix. Les aires de répartition des espèces les moins rhéophiles : Polycelis nigra et Dugesia lugubris dépendent du relief; ces espèces occupent les régions de basses plaines à l'ouest d'une ligne Aix-Marseille

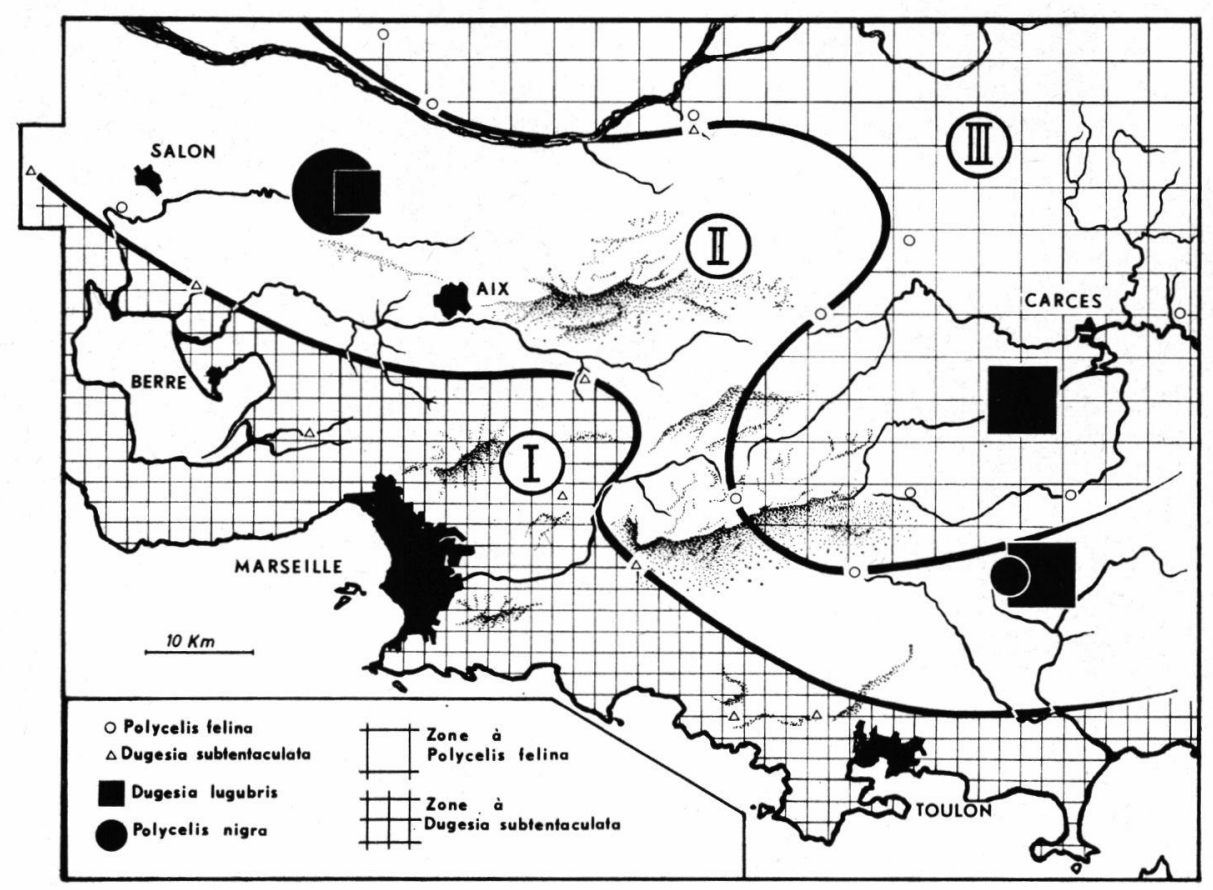

Fig. I. - Zones de répartition des Planaires Triclades d'eau douce en Provence occidentale. 
et les environs d'Hyère. Dugesia lugubris peuple seule la vallée de l'Argens.

La région prospectée peut donc être divisée en trois zones de répartition.

- La zone I correspond à l'aire de répartition de Dugesia subtentaculata et renferme en outre : Dendrocoelum lacteum, Dugesia lugubris, Polycelis nigra et Dugesia tigrina.

- La zone II, du fait de son relief, est subdivisée en :

- une partie centrale accidentée (massifs de la Sainte-Baume et de la Sainte-Victoire), renfermant l'espèce la plus rhéophile : Dugesia gonocephala et une espèce ubiquiste: Dendrocoelum lacteum;

- deux parties latérales (est et ouest) moins accidentées, habitées par les deux espèces précédentes et par les espèces limnophiles, Dugesia lugubris et Polycelis nigra.

- La zone III correspond à l'aire de répartition de Polycelis felina à laquelle sont associées : Dugesia gonocephala, Dugesia lugubris et Dendrocoelum lacteum.

\section{Zonation (étagement de l'habitat).}

\section{A) Confirmation du schéma classique.}

Le schéma classique ne pourrait trouver son application qu'au sein d'un cours d'eau de longueur suffisante qui prendrait naissance dans la zone III et déboucherait dans la zone I. Le seul exemple qui nous soit donné est une rivière dont la source est située à la limite sud de la zone III : le Gapeau. La plupart des cours d'eau de Provence prennent naissance dans la zone II; ils ne renferment donc pas Polycelis felina. Ou bien ils naissent dans la zone III et leur parcours est alors infléchi vers l'Est par la barrière que constituent les massifs côtiers, ils n'hébergent donc pas Polycelis nigra.

- Cas des cours d'eau à orientation Est : Issole, Caramy, Argens, jusqu'au lac de Carcès.

Ils drainent les eaux des versants orientaux des massifs de la Sainte-Baume et de la Sainte-Victoire avant de se jeter dans le lac de Carcès. Ces trois rivières prennent naissance dans la zone III. Leur partie amont est habitée par Polycelis felina et Dugesia gonocephala; la partie moyenne n'est occupée que par Dugesia gonocephala à laquelle se joint Dugesia lugubris à la partie inférieure. Comme dans les autres cours d'eau de Pro- 
vence, Dendrocoelum lacteum ne semble pas avoir une place définie dans la zonation (fig. $2 \mathrm{~B}$ ).

- Cas des cours d'eau à orientation Sud: Le Gapeau, l'Huveaune.

Le Gapeau prend sa source au pied du massif de la SainteBaume et se jette dans la rade d'Hyères après un cours de $50 \mathrm{~km}$. Cette rivière est habitée par cinq espèces de planaires qui s'étagent selon le schéma classique (fig. $2 \mathrm{C}$ ).

L'Huveaune chemine entre les massifs de la Sainte-Baume et d'Allauch avant de se jeter à Marseille dans l'anse du Prado. Seul le cours supérieur, peuplé par Dugesia gonocephala, n'est pas pollué.

- Cas des cours d'eau à orientation Ouest : l'Arc, Touloubre.

Ils cheminent dans une région plate. Dans sa partie amont, la Touloubre n'est peuplée que par Dugesia lugubris qui occupe

A

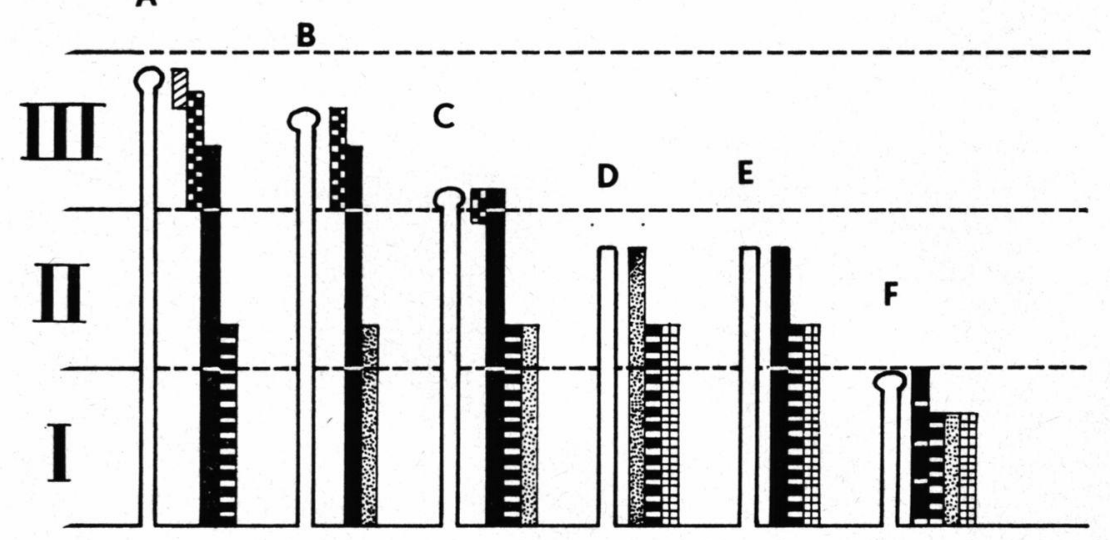

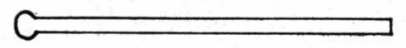

बापाएण C.alpina

a.r.telina

D. gonocephala

\section{source + ruisseau}

D.lugubris

姍册 D.tigrina

D. subtenfaculata

\section{IIIII P. nigra}

Fig. II. - Etagement de l'habitat chez les Planaires d'eau douce. A : schéma classique de l'étagement de l'habitat des Planaires d'eau douce dans les cours d'eau européens; B : répartition dans l'Issole, le Caramy et l'Argens; C : répartition dans le Gapeau; D : répartition dans la Touloubre; E : répartition dans l'Arc; F : répartition dans les ruisseaux de faible longueur. 
la place de Dugesia gonocephala. Dans sa partie aval, Polycelis nigra et Dugesia tigrina se joignent à Dugesia lugubris. Dendrocoelum lacteum n'occupe pas une place déterminée dans ce cours d'eau (fig. $2 \mathrm{D})$.

L'Arc est une rivière plus rapide que la précédente. Dugesia gonocephala reprend sa place loul le long du cours d'eau principal; Polycelis nigra et parfois Dugesia lugubris (la Jouine) peuplent la partic terminale (fig. $2 \mathrm{E}$ ).

La zonation des planaires dans les cours d'eau est, en grande partie, confirmée par la répartition des trois espèces du schéma classique présentes parmi les quatre espèces qui s'étagent dans notre région.

Polycelis felina, lorsqu'elle esl présente, occupe loujours la partie amont du cours d'eau, mais lorsque les conditions thermiques l'excluent, sa place est prise par Dugesia gonocephala. Cette dernière espèce occupe le cours moyen; mais lorsqu'elle est absente, sa place n'est pas occupée par la planaire qui lui succède dans la série type, mais par une espèce qui ne figure pas dans la séquence normale : Dugesia lugubris.

Polycelis nigra habite seule ou en compagnie de Dugesia lugubris, la partie aval; lorsqu'elle est absente, Dugesia lugubris occupe seule cette zone.

\section{B) INVERSION APPARENTE DE LA ZONATION.}

Celte inversion se produit dans le cas où deux sources l'une limnocrène, l'autre rhéocrène (située en aval de la précédente), sont collectées par le même ruisseau. Deux exemples : les sources de Saint Paul-lez-Durance et celles de Grans (fig. 3 ).

\section{- Cas de Saint Paul-lez-Durance.}

La source limnocrène en amont est habitée par Polycelis nigra, la source rhénocrène en aval par Polycelis felina. Dugesia subtentaculata peuple le cours d'eau collecteur; Dendrocoelum lacteum occupe les trois biotopes. Les planaires du genre Polycelis sont sélectivement réparties dans ce milieu qui pourtant est ouvert aux deux bouts. Polycelis nigra ne sort jamais de la source limnocrène alors que Polycelis felina migre souvent en dehors de la source rhéocrène et remonte le ruisseau jusqu’à quelques dizaines de mètres de la source amont sans y pénétrer. Faut-il, pour expliquer cette particularité de la répartition, faire intervenir certains facteurs sélectifs tels la température, le courant ou la chimie des eaux? Il est peu probable que la température soit à l'origine 
de leur séparation spatiale, puisque les deux sources ont des températures assez semblables et assez constantes : $14-15^{\circ}$ pour la source limnocrène, $13-14^{\circ}$ pour la source rhéocrène. Les analyses chimiques effectuées ne révèlent aucune différence; seul le courant varie. On peut penser que le courant nul de la source limnocrène représente un facteur limitant pour la progression de Polycelis felina. A l'opposé, Polycelis nigra ne sort jamais de son biotope alors que l'existence d'un courant faible, tel celui du ruisseau collecteur, ne constitue pas une barrière à sa migration puisque j'ai pu la récolter dans d'autres cours d'eau présentant des caractéristiques semblables (Jouine, Cadière, etc...) et même dans une rivière au courant plus fort comme le Gapeau. On est amené à interpréter la répartition de ces deux espèces, à petite échelle, par des relations de concurrence alimentaire ou territoriale.

\section{- Cas de Grans.}

Ici aussi deux sources sont drainées par un même ruisseau. La première constitue un bassin d'eau calme, elle est peuplée par Polycelis nigra et Dugesia lugubris. La seconde est constituée d'une eau courante comme le ruisseau qui résulte de la jonction des deux sources. La source rhéocrène et le ruisseau collecteur sont habités par Polycelis felina. Aucune des deux espèces congénériques vivant dans cette station ne s'aventure dans l'habitat occupé par l'autre. Les conditions physico-chimiques de ces biotopes sont pourtant les mêmes. Seul le courant varie; mais, comme dans l'exemple précédent, il ne constitue pas un obstacle à la migration de Polycelis nigra.

\section{C) Problèmes posés par un cours d'eau de longueur réduite.}

Les cours d'eau de faible longueur sont issus de source, ils possèdent donc des températures assez stables et relativement élevées pendant toute l'année : Saint Paul-lez-Durance, Saint-Pons, la Cadière qui possèdent des températures de l'ordre de 13 à $15^{\circ}$, la Durançole dont la température varie de 18 à $20^{\circ}$, etc...

Dugesia subtentaculata peuple tout le trajet, de la source à la limite inférieure du ruisseau. Lorsque le cours d'eau est suffisamment lent sa partic aval est occupée par les espèces limnophiles Polycelis nigra et Dugesia lugubris (la Cadière, Mouriès) toujours en compagnie de Dendrocoelum lacteum (fig. 2 F).

D) Problèmes posés par la présence de Dugesia tigrina.

Dugesia tigrina est présente dans tous les cours d'eau qui se jettent dans les étangs de Berre et de Bolmon ainsi que dans 


\section{Source limnocrène} \\ SAINT PAUL - LEZ - DURANCE}
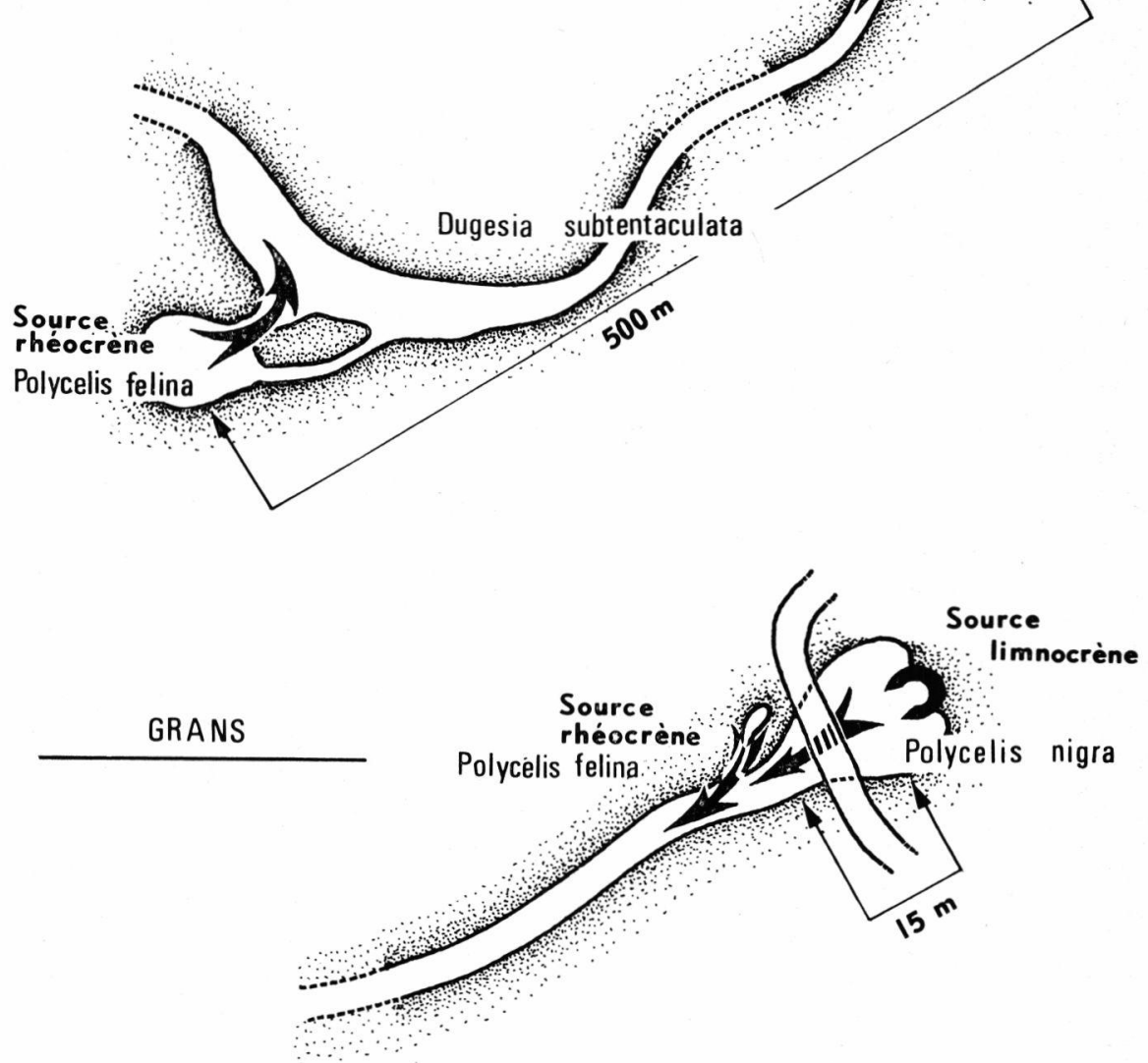

FIG. III. - Inversion apparente de la zonation dans deux stations.

une station d'un cours d'eau côtier, en partie temporaire : le Destel, situé dans le Var.

La présence de cette espèce dans notre région pose un problème qui n'a pas encore trouvé de réponse (LÉGIER $1969 \mathrm{~b}$ ). Avant septembre 1967, je n'avais remarqué sa présence dans aucun des cours d'eau de la région; depuis on la trouve dans la Touloubre, dans la Durançole, dans l'Arc, dans la Cadière et dans le Destel, 
alors qu'au cours d'une étude réalisée sur ce dernier ruisseau en 1961, GIUdicellr et LÉonide ne l'avaient pas récoltée.

Un cas semblable s'est produit dans le Lez et dans la Mosson, rivières côtières des environs de Montpellier, où Tuzet et DeL Perrugia (1967) ont récolté cette espèce alors que quelques années auparavant ni VANDEL, ni BENAzZi ne l'avaient signalée.

Une étude quantitative effectuée sur la Durançole (LÉGIER 1969 a), a montré que la présence et la multiplication rapide de Dugesia tigrina dans le biotope à Dugesia subtentaculata a entraîné une diminution du nombre des individus de cette dernière espèce. Le nombre des individus de Dugesia tigrina a ensuite diminué pour arriver à un équilibre correspondant à une cohabitation d'un petit nombre d'exemplaires de ces deux espèces. Comme l'ont signalé les deux auteurs montpelliérains, la coexistence entre Dugesia tigrina et Dugesia subtentaculata paraît difficile.

\section{CONCLUSION}

La répartition des espèces de planaires triclades d'eau douce en Provence occidentale nous a conduit à découper cette région en trois zones. On note le remplacement des espèces du nord au sud : Polycelis felina est l'espèce la plus septentrionale; Dugesia gonocephala occupe la partie centrale de cette région tandis que la zone littorale héberge à la fois Dugesia subtentaculata et Dugesia tigrina. Les parties est et ouest du territoire prospecté sont habitées par Polycelis nigra et Dugesia lugubris, la première domine à l'ouest, la seconde domine à l'est (fig. 1).

Ce schéma de répartition horizontale se retrouve à une échelle plus réduite, celle du ruisseau, avec le remplacement des espèces depuis l'amont vers l'aval. Cette étude confirme et précise le schéma classique de la zonation des planaires. L'ordre de la séquence ne subit pas d'inversion sauf dans certaines stations du crénon où des particularités topographiques permettent la présence de Polycelis nigra, espèce qui ordinairement peuple la portion aval des ruisseaux.

Il convient toutefois de faire intervenir un facteur qui, en Provence, parâit déterminer la présence, aux côtés de Polycelis nigra, de Dugesia lugubris, espèce qui ne figurait pas dans le schéma classique. Lorsque la mase d'eau véhiculée est importante, Dugesia lugubris est toujours présente (région est du territoire); on la rencontre aussi dans certains ruisseaux au débit plus faible. Mais à l'opposé, Polycelis nigra est à peu près toujours absente des cours d'eau à fort débit; par contre, sa présence est constante 
dans les petits ruisseaux (région ouest du territoire). Dans les cours d'eau importants, au courant lent, Dugesia lugubris occupe la place de Dugesia gonocephala dans la séquence.

Enfin, lorsqu'un ruisseau prend naissance dans une zone climatique chaude, c'est Dugesia subtentaculata qui l'habite et Polycelis nigra se cantonne dans la partie aval.

Le schéma classique de la zonation des espèces d'amont en aval, sur le trajet des cours d'eau, est donc en grande partie confirmé pour la Provence occidentale. Cependant, en raison d'une part de la situation climatique de la région prospectée, et d'autre part de certaines particularités topographiques, ce schéma se trouve amputé dans sa première partie (absence de Crenobia alpina et quelquefois de Polycelis felina) et modifié dans sa troisième partie (présence à peu près constante de Dugesia lugubris dans les cours d'ean à fort débit).

Le problème de l'insertion de Dugesia tigrina dans le schéma reste à préciser.

\section{RÉSUMÉ}

Le présent travail traite de la répartition géographique et de l'étagement de l'habitat de sept espèces de planaires récoltées en Provence occidentale.

Ces espèces possèdent chacune une aire de répartition plus ou moins étendue. La détermination de ces aires permet de diviser la région prospectée en trois zones de répartition.

La séquence altitudinale des espèces de planaire sur le trajet des cours d'eau se fait, en général, selon le schéma classique observé en Europe. Mais, selon la situation des cours d'eau à l'intérieur de ces zones et du fait de certaines particularités topographiques, cette succession se trouve plus ou moins amputée ou modifiée.

\section{SPATIAL DISTRIBUTION AND STRATIFICATION OF THE BIOTOPES OF FRESH-WATER PLANARIANS IN WEST PROVENCE}

This investigation deals with the spatial distribution and the stratification of seven species of Planarians collected in West Provence.

Each species possesses its own spatial area. The extention of these specific areas suggests a division of the investigated region in three zones of distribution.

Generally, the altitudinal sequence of planarian species in a stream confirms the classic observations made in Europ. But, according to the situation of the streams in these zones and consequently of certain topographic particularities, this succession may be modified.

\section{HORIZONTALE UND VERTIKALE VERBREITUNG VON SUSSWASSERPLANARIEN IN DER WEST PROVENCE}

Diese Arbeit behandelt die geographischen Verbreitung von sieben Planarien und die Abfolge ihrer Habitate gemä $B$ der Höhenlage. Jede dieser Arten hat ein mehr oder weniger weites Verbreitungsgebiet. Die 
Bestimmung der Verbeitungsgebiete erlaubt eine Unterteilung der untersuchten Region in drei Verbreitungszonen. Die Artenabfolge aufgrund der Höhenlage entspricht dem klassischen für Europa beobachteten Schema. Jerloch aufgrund der Lage Fließgewässer innerhalb dieser Zonen und aufgrund einiger topographischer Eigentümlichkeiten ist diese Abfolge fragmentarisch oder modifiziert.

\section{TRAVAUX CITES}

BeAUchamp (R. S. A.). 1932. - Some ecological factors and their influence on competition between stream and lake-living Triclads. J. Anim. Ecol., 1 : 175-190.

Beauchamp (R. S. A.) et Ullyott (P.). 1932. - Competitive relationships between certain species of fresh-water Triclads. J. Ecol., 20 : 200208.

Chander (C. M.). 1966. - Environmental factors affecting the local distribution and abondance of four species of stream-dwelling Triclads. Dissert. Abstr. U.S.A., 26, II, 6971. Res. thèse, Indiana Univ.

Dанм (A. G.). 1958. - Taxonomy and Ecology of five species groups in the family Planariidae (Turbellaria Tricladida Paludicola). Thèse Sc. Lund. Malmö : 1-241.

Fiasson (S.). 1964. - Recherches écologiques sur la faune d'une rivière de la région lyonnaise : l'Yzeron. Thèse Sc. Lyon : $182 \mathrm{p}$.

Giudicelli (J.) et Leonide (J. C.). 1961. - Note préliminaire sur l'hydrobiologie d'un ruisseau de la région toulonnaise : le Destel. Ann. Fac. Sc. Marseille, $31: 137-142$.

Legier (P.). $1969 a$. - Etude écologique des Planaires Triclades d'eau douce en Provence occidentale. Thèse Doctorat Spécialité, Marseille : 162 p.

Legier (P.) 1969 b. - . Présence dans les Bouches-du-Rhône de la Planaire Dugesia tigrina (Girard). Bull. Mus. Hist. Nat. Marseille, XXIX : 73-80.

Pattee (E.). 1966. - Coefficients thermiques et écologie de quelques Planaires d'eau douce: I. Tolérance des adultes. Annls. Limnol., $2,3: 469-475$.

- 1968 - II. Tolérance de Dugesia gonocephala. Annls. Limnol., 4, $1: 99-104$.

- 1969 - III. La reproduction de deux espèces montagnardes. Annls. Limnol., 5, 1 : 9-24.

- 1970 - IV. La reproduction de Dugesia gonocephala. Annls. Limnol., 6,3 : 293-304.

Pattee (E.) et Bournaud (M.). 1970. — Etude expérimentale de la rhéophilie chez les Planaires Triclades d'eau courante. Schweiz. Zeitsch. für Hydrol., 32, 1 : 181-191.

Tuzet (0.) et Der. Perrugia (A. M.). 1957. - Présence de la Planaire américaine Dugesia (Euplanaria) tigrina aux environs de Montpellier. Vie Milieu, 8, 1.

Valldant (F.). 1968. - Sur le choix des espèces indicatrices pour une zonation des eaux courantes. Trav. Lab. Hydrobiol. Pisc. Univ. Grenoble, 57-58, 7-15.

Volgt (W.). 1895. - Planaria yonocephala als Eidringling in das Verbreitungsgebiet von Planaria alpina und Polycelis cornuta. Zool. Jb., Abt. Syst. Ukol. Geogr., 8 : 131.176. 